repair, and epigenetic regulation. Mutations in LMNA gene, which encodes for lamin A protein, cause a large variety of human diseases, known as laminopathies, including muscular dystrophies and progeroid syndromes. Phosphatidynositol4,5-bisphosphate (PIP2) is a well described product of the phosphoinositol signalling and it has an important role in splicing and transcription. Here we show that PIP2 is in a complex with lamin A, together with Nuclear myosin 1 (NM1), and lamin A phosphorylation status appears to be implicated in the anchoring of other proteins to the complex, as well as in the movement of lamin A from the nuclear envelope to the nucleoplasm. Thus, we are characterizing this complex using several biochemical and microscopy methods. From our preliminary data we conclude that some Lamin A phosphorylations might be important for PIP2-dependent interactions of lamin A, important for nuclear functions.

We would like to thank to the Grant Agency of the Czech Republic (17-09103S, 16-03346S and 15-08738S) for their financial support to this project. We would also like to thank for the institutional support due to the long-term conceptual development of the scientific organization (RVO: 68378050). The microscopy work was performed at the Microscopy Centre, Institute of Molecular Genetics AS CR, supported by the MEYS CR (LM2015062 Czech Bioimaging).

doi: http://dx.doi.org/10.7124/bc.0009B7

\section{From 3D-Genome Organisation to Cellular and Supra- cellular Morphogenesis}

\section{Klaus Scherrer}

Institut J. Monod, CNRS and Univ. Paris Diderot, klaus.scherrer@ijm.fr
The molecular 3D-model of the "Unified Matrix Hypothesis" (UMH) proposed that the eukaryotic DNA may be organised in a 3D-network and genomic domains, to be transcribed in specific sectors of the nucleus defining "chromosome territories"(1). It generalised by analogy for normal cells the pattern of Ectopic Pairing observed since 1948 in drosophila salivary glands, that links the 4 polytene chromosomes and the nucleolus into a 3D-genomic network. In normal cells where DNA is flexible, an analogous system might operate able to link directly distant sites within and between chromosomes. This putative model at the basis of nuclear architecture and morphogenesis was confirmed by the publication of the $3 \mathrm{D}$ structure of the yeast genome (2) and more recently extended to eukaryotic cells in general (2). The original model of the $\mathrm{UMH}$ also proposed a logical link between cellular and supra-cellular morphogenesis. The 3D-DNA chromatin network organises the nuclear architecture which can be modified back and forth by conversion of hetero- and euchromatin, or by transposition of mobile DNA elements as SINES and LINES or other «jumping genes». In undifferentiated stem cells and de-differentiated cells, having lost their cytoplasm by «shedding», the nucleus occupies most of the cellular volume. In differentiation, it is reduced by retraction of its membrane from the periphery to the center, ending up fully picnotic in terminal differentiation of, e.g. red cells,. In this process, nuclear space becomes cytoplasm leaving behind, possibly, some of its organisation. In the cytoplasm localised synthesis of specific proteins is based on prior 3D-sorting of mRNA; the various types of cytoskeleton are instrumental 
in this process, in particular the IF and the tubulin network. The latter is organised from the centrosome positioned at the nuclear membrane. - Prior to cell division the centrosomes divide and move to opposite poles of the cell from where the spindle is formed in mitosis; this determines the direction of cellular division in space. However, prior to daughter cell formation the spindle may change its position within the cellular space. Programs of spindle (re-)orientation define the direction of subsequent cell divisions; combined with allowed numbers of divisions this fixes primary morphogenesis, prior to cell-cell interaction and selective apoptosis. Spindle re-orientation happens at critical steps of differentiation and morphogenesis and is based on the internal topological organisation of the mitotic cell. Thus, 3D organisation of the human genome, transcripts and gene expression may link DNA polymorphism and supra-cellular morphogenesis in individuals, as a paradigm of, e.g., definition of facial patterns obviously linked to genetics.

\section{Workshop III DNA REPLICATION, REPAIR AND CANCER}

doi: http://dx.doi.org/10.7124/bc.0009B8

\section{Unrepairable analogous of nucleotide excision repair substrates as a potential anti-cancer drugs}

Alexey N Evdokimov*1, Evgeniya V Dolgova $^{* 2}$, Alexey A. Popov ${ }^{1}$, Irina O. Petruseva $^{1}$, Sergey S Bogachev*2, Olga I Lavrik*1,3

* These authors contributed equally to this work

${ }^{1}$ Institute of Chemical Biology and Fundamental Medicine Siberian Branch of the Russian Academy of Sciences, Novosibirsk 630090, Russia; ${ }^{2}$ Institute of Cytology and Genetics, Siberian Branch of the Russian Academy of Sciences, Novosibirsk 630090, Russia; ${ }^{3}$ Novosibirsk State University, Novosibirsk 630090, Russia

an_evdokimov@mail.ru

In the previous studies we have demonstrated that DNA with the bulky Fap-dC derivative is a hardly repairable substrate for the cellular
NER system [1]. Such type of compounds could be of particular interest as possible selective inhibitors of the NER system, considerably reducing the potency of DNA repair due to competitive immobilization of protein factors involved in this process. Tumor-initiating stem cells (TISCs) which are capable to internalize exogenous DNA $[2,3]$ could be the potential target for such synthetic analogues of NER substrates. In the current work the process of DNA internalization was considered as an approach to deliver the model DNAs into TISCs in order to reduce the reparative potential of cancer cells. Methods: Enzymatic DNA synthesis, PCR, RT-PCR, NER-competent cell extract preparation, in vitro NER assay, lab animals breeding, fluorescence microscopy, laser scanning microscopy. Results: 756 bp PCR product containing bulky photoactivable $\mathrm{dC}$ adducts inhibits the nucleotide excision repair system. The Krebs-2 ascites cells were 